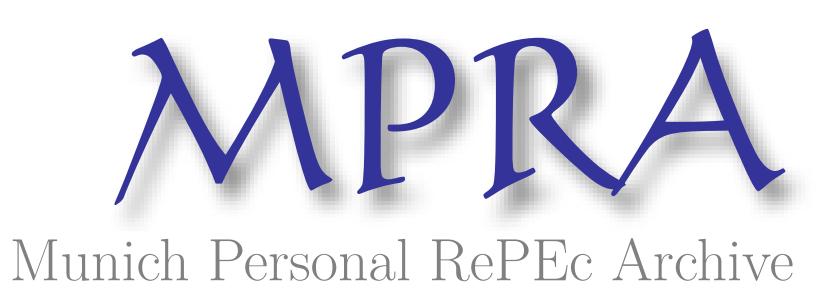

\title{
Downside Risk for European Equity Markets
}

Cotter, John

2004

Online at https://mpra.ub.uni-muenchen.de/3537/

MPRA Paper No. 3537, posted 12 Jun 2007 UTC 


\title{
Downside Risk for European Equity Markets
}

\author{
JOHN COTTER \\ University College Dublin
}

\begin{abstract}
This paper applies extreme value theory to measure downside risk for European equity markets. Two related measures, value at risk and the excess loss probability estimator provide a coherent approach to optimally protect investor wealth opportunities for low quantile and probability combinations. The fat-tailed characteristic of equity index returns is captured by explicitly modelling tail returns only. The paper finds the DAX100 is the most volatile index, and this generally becomes more pronounced as one moves to lower quantile and probability estimates.
\end{abstract}

Keywords: risk estimation, extreme value theory, fat-tailed equity returns.

\author{
Address for Correspondence: \\ Dr. John Cotter, \\ Centre for Financial Markets, \\ Department of Banking and Finance, \\ Graduate School of Business, \\ University College Dublin, \\ Blackrock, \\ Co. Dublin, \\ Ireland. \\ E-mail. john.cotter@ucd.ie \\ Ph. 00-353-1-7168900
}




\section{Downside Risk for European Equity Markets}

\section{INTRODUCTION}

Recently downside risk has become a highly debated topic, recognising the need for appropriate risk management practices given that systematic risk cannot be eliminated. ${ }^{1}$ Amongst the methods applied are mean variance analysis (portfolio theory) and Value at Risk (VaR). The use of these approaches aims to control downside risk so as to maximise investor wealth opportunities. Portfolio theory examines a risk return trade-off for different combinations of assets in order to control risk for given return levels. Similarly, VaR focuses on a maximum level of loss that investors would be willing to incur given the returns distribution. This quantile measure gives investors specific information on the degree of downside risk at a number of probabilities and horizons (for example, a single VaR statement might indicate that an investment's losses should not exceed 5\% for a 10 day holding period and at the $95 \%$ confidence level). ${ }^{2}$ In a risk management context, the uniform aim of both approaches is to control the possible losses that the investor may encounter.

This paper uses a semi-parametric methodology, extreme value theory, to quantify tail behaviour for European equity indexes in a VaR framework. Alternatively, time series analysis has focused on the full distribution of returns testing the random walk and mean reversion hypotheses (Gallagher, 1999). Relating to VaR measurement, a number of studies have examined equity returns series to determine their true underlying distributional properties (Ghose and Kroner, 1995; and Poon 
and Taylor). Diverging support is offered regarding a number of separate hypotheses including $\mathrm{ARCH}$ and stable paretian related distributions. Extreme value theory nests both hypotheses thereby providing a unique approach to distinguish between the alternative distributions. Nevertheless, all the studies agree that the financial data analysed exhibits fat tails relative to the normal distribution. This characteristic can lead to problems in $\mathrm{VaR}$ computations if one assumes a distribution that does not exactly fit the data under analysis. In particular, assuming gaussian properties for equity index returns leads to an underestimation of the tail index, and consequentially their counterparts, the VaR measures. Making use of extreme value theory that specifically models tail behaviour only, and allowing for the case of fat tails gives greater precision to the lower quantile VaR estimates (Diebold et al., 1998).

Furthermore, a series' of papers have focused on various issues regarding VaR computations (Danielsson and DeVries, 1997a, 1997b, 2000; Kearns and Pagan, 1997; Duffie and Pan, 1997; Venkataraman, 1997; and Loretan, and Phillips, 1994). The findings offer further support in the use of extreme value theory as the most appropriate method in calculating VaR estimates; the Hill (1975) statistic as being the optimal tail estimate; and that the properties of extreme returns are unlike the full series of returns. Moreover, VaR theoretically suffers from an inability to measure risk beyond the chosen quantile (Artzner et al., 1999). Thus, to provide a coherent description of downside risk there is a need for a measure that goes beyond the VaR. This paper outlines and measures a new statistic, the excess loss probability estimator for examining this issue. This estimator measures the probability of having losses in excess of VaR quantiles. 
The outline of the paper is set out as follows. Section II deals with theoretical and

methodological issues. Initially a description of related VaR measures are given. This is followed by a debate on the relative merits of extreme value theory. Section II is concluded with a presentation of the main theoretical and methodological findings of extreme value theory. Then a description of the data is given in the third section, showing preliminary statistics for the indexes analysed, as well as a snapshot of the extreme returns. Results are presented and discussed in section IV. Finally, conclusions are detailed in section V.

\section{THEORITICAL AND METHODOLOGICAL ISSUES}

\section{Value at Risk (VaR)}

Value at Risk $(\mathrm{VaR})$ is a statistical measure of (downside) risk that estimates the maximum loss that may be experienced by a portfolio (index) over a given period with a given confidence level. Taking a random variable $\mathrm{R}$, representing the set of index returns, with distribution function $\mathrm{F}$ detailing all positive and negative returns over a prespecificied time period, its risk can be defined by a VaR estimate. The VaRq is the $\mathrm{q}^{\text {th }}$ quantile of the distribution of negative returns

$\operatorname{VaRq}=-\mathrm{F}^{-1}(\mathrm{q})$

where $\mathrm{F}^{-1}(\mathrm{q})$ is the quantile function and is the inverse function of $\mathrm{F}$.

Graphically, the VaRq is represented in figure 1 with the quantile cut-off point VaR (R) measuring the maximum loss for that given quantile.

INSERT FIGURE 1 HERE 
However the VaR measure cannot be considered as a panacea for risk management estimation. While (1) gives a measure for the amount of possible downside risk exposure upto the extreme negative return, $r$, it has been criticised for not describing the losses in excess of the VaRq that an investor can incur (Artzner et al., 1999). We would expect a priori that a risk averse investor is similarly illdisposed (or even more so) to losses in excess of the VaR as they are to the measure itself. For instance, considering figure 1 the $\mathrm{VaR}$ estimate does not tell us anything about returns further out in the tail, those values that can be considered as the Excess Loss (ELp) of the VaR. Thus to provide a coherent picture of downside risk we should also examine the probability of having excess losses greater that a VaR threshold by focusing on the probability mass to the left of $\mathrm{VaR}$ in figure 1 . Formally this is given as

$\operatorname{ELp}=\mathrm{P}[\mathrm{R} \mid \mathrm{R}>\mathrm{VaRq}]$

for extreme (negative) returns.

The most controversial element of the Riskmetrics system is the assumption that the unconditional distribution of financial returns follows a normal distribution. However one of the main stylized facts for financial time series is the rejection of normality (see findings later for our equity indexes). Moreover, the empirical features all to some degree indicate volatility clustering, and there is evidence that this characteristic of financial time series impinges on the efficiency of the tail estimates (Duffie and Pan, 1997). In particular, parametric approaches including portfolio theory (variance-covariance analysis) underestimate extreme returns, regardless of whether they are located in the upper or lower tail of the distribution. This is a major problem for Value at Risk measures where the potential tail losses 
estimated are greater for the true data generating processes, than that of their gaussian counterpart. This conclusion can be clearly seen for any quantile by focusing on the downside tail returns given in figure 2. Here, the empirical distribution of equity index returns (shaded curve), following a fat tailed process, is plotted against the normal distribution (solid curve), following a relatively thin tailed process. At all times, the normal distribution underestimates the downside tail behaviour vis-à-vis the fat tailed process.

\section{INSERT FIGURE 2 HERE}

Furthermore, a case study will emphasise the magnitude of the underestimation issue. For instance a clear example of the tails from a normal distribution underestimating downside risk is the case of the stock market crash, 19 October 1987. Here, most international equity markets showed actual losses in excess of 10\%. However, under gaussian assumptions, such an occurrence would only occur once every 5900 years (Venkataraman, 1997). Obviously empirical evidence is contrary to this forecast showing greater losses being recorded in a shorter time frame, for example, the occurrence of the crash of1929. A similar conclusion can be made for any large negative loss in any asset market. The most common measure actually applied in relation to tail densities is the centred kurtosis statistic, the fourth moment of a distribution. For the normal distribution, this estimate takes on a value of 0 , whereas in the presence of platykurtosis, thin tails, the value is less than 0 . Leptokurtosis is consistent with relatively fat tails relative to the gaussian case, and the centred kurtosis values are greater than 0 . 


\section{Debate on Extreme Value Theory:}

As we have seen with gaussian estimation of tail values the primary empirical consideration in using the $\mathrm{VaR}$ approach is to apply the most efficient estimation technique. This paper picks the extreme value theory (see Leadbetter et al., 1983, for a comprehensive discussion) at the expense of the more traditional methods of the variance-covariance approach, historical simulation and Monte Carlo simulation. Its approach lies in calculating a lower tail percentile from the distribution of returns for some prespecified time period. This is equivalent to focusing on the large losses of an investment, that is, the maximum downside of risk.

There has been considerable debate of the relative strengths and weaknesses of using extreme value theory in examining tail behaviour (Diebold et al., 1998). Both theoretical and practical issues have been the focus of discussion. This paper briefly presents a synopsis of these and examines those of relevance to the empirical application at hand. To begin, turning to the strengths, an application of extreme value theory does not suffer from model risk in comparison to the variance covariance approach (standard portfolio theory) and Monte Carlo methods. These alternative approaches examine probabilities for the full distribution of returns, whereas the actual focus of attention, the tail values are explicitly modelled separately by extreme value theory. Thus for instance, we always end up with underestimation of tail behaviour by applying the variance covariance approach, and result in inappropriate tail estimates with Monte Carlo simulation of alternative full distributions such as the student-t process (see Cotter, 2001; for an illustration). This bias results from modelling the centre of the distribution where no such 
analysis is required. Alternatively simulation has been used to examine different (worse case) scenarios. These however are subjectively defined and unlike the application of extreme value theory, they do not define an objective likelihood function.

A key addition that extreme value theory makes is that it removes the need for making assumptions as to the exact distributional form of the data under analysis. It separates out three types of distribution of which financial returns belong to the fat tailed classification. This is because under extreme value theory all fat tailed distributions have the same limit law and thus the correct distributional form is not a primary concern. Briefly, this limit law induces an identical feature in fat tailed realisations as they exhibit a regular variation property. Feller (1971) found that this regular variation at infinity property is the sufficient and necessary property for fat tailed distributions to hold. It implies that not all moments of the fat tailed distribution are bounded unlike other types of distributions such as normality.

Furthermore extreme value estimates can easily be extended from a certain observation frequency, for example daily to weekly estimates (see Jansen et al., 2000; for an illustration). This extension is similar to the scaling law of normality where lower frequency estimates follow a square root of time rule. Both approaches do not require any further estimation and there is no need for additional observations measured at different frequencies. Thus for instance, daily extreme value estimates can easily be scaled up for weekly estimates without estimating weekly returns. This is particularly advantageous in relation to the tail estimation 
procedure that is applied in this study, which can be impaired for smaller sample sizes, that is by examining data sets at lower frequencies. ${ }^{3}$

Finally, our semi-parametric tail estimation procedure can be extended for out-ofsample periods (see empirical illustration later). This allows us to give very low probability tail estimates and thus dominate the historical simulation approach that is restricted to probabilities $(1 / \mathrm{n})$ within the sample size, $n$. Thus the downside risk measures presented go beyond the sample size estimated, for example using 5 years of data to obtain downside risk forecasts for a 10 year period. Thus, these out-ofsample forecasts remove the data size restrictions that impair many empirical studies.

Notwithstanding the attractive features of extreme value theory, there are a number of potential weaknesses of the approach. First, the statistical theory on the properties of the finite-sample Hill index (our downside tail estimator) is as yet not fully developed although it is consistent and asymptotically normal for infinite samples. Furthermore, the Hill index is used indirectly in risk management estimation as it is extreme probability and quantile estimates that are presented. These estimates are non-linear functions of the Hill index, and as a consequence of any poor statistical properties of this measure, would themselves suffer suboptimal statistical features. Second, much of extreme value theory has been presented for an identical and independently distributed variable, in contradiction to the volatility clustering inherent in financial returns. However, the theory has been extended to deal with a stationary case (see Leadbetter et al., 1983). This property is accepted for the first difference of financial price series, namely their returns. Finally, it is 
generally assumed that financial returns are in the maximum domain of attraction of a Fréchet distribution implying that the tail follows a power law. However, financial variables such as those relating to credit risk estimation do not have fat tailed features. Thus it is necessary to first ensure that the stock index returns analysed are fat tailed by examining their centred kurtosis statistic and the value of the Hill tail index.

\section{Extreme Value Estimation:}

The key addition that extreme value theory makes to downside risk analysis is that it removes the need for making assumptions as to the exact distributional form of the data under analysis. The asset returns series may follow any fat tailed distribution such as a stable paretian or mixtures of normals, but at the limit they all converge to the same underlying distribution. As the same limit law applies, the correct distributional form of the asset returns is not a primary concern. For the fattailed case, returns are assumed to belong to the maximum domain of attraction of the Fréchet distribution. This result follows a similar role as the central limit theorem and normality but here asymptotically the returns converge to the fat tailed distribution. ${ }^{4}$ To overcome the lack of an exact fit for the finite equity index returns this study uses a semi-parametric tail estimator, the moments based Hill estimator.

Formally, taking a sequence of strictly stationary returns $\left\{R_{1}, R_{2}, \ldots, R_{n}\right\}$ that may, but not necessarily be identical and independently distributed (Leadbetter et al., 1983), and that has a distribution function F. As we are dealing with large negative 
returns, we can examine the minima $\left(\mathrm{M}_{\mathrm{n}}\right)$ of a sequence of $\mathrm{n}$ random variables rearranged in ascending order where

$\mathrm{M}_{\mathrm{n}}=\operatorname{Min}\left\{\mathrm{R}_{1}, \mathrm{R}_{2}, \ldots, \mathrm{R}_{\mathrm{n}}\right\}$

where $M_{n}$ is an order statistic

and

$\mathrm{P}\left\{\mathrm{M}_{\mathrm{n}} \leq \mathrm{r}\right\}=\mathrm{P}\left\{\mathrm{R}_{1} \leq \mathrm{r}, \ldots, \mathrm{R}_{\mathrm{n}} \leq \mathrm{r}\right\} \quad=\mathrm{F}(\mathrm{r}) \quad-\infty<\mathrm{r}<\infty$

The random variables of interest in this analysis, the negative returns, are located at the tail of the distribution, F(r). For example, the VaR measures the amount of possible loss exposure upto the extreme return, r. Losses in excess of this extreme return can be generated, and related measures of the probability of exceeding the extreme return, $r$, are given by Excess Loss (ELp) estimators for a range of possible losses.

As stated we model our fat tailed returns to be in the maximum domain of attraction of the Fréchet distribution. In fact, extreme value theory examines three alternative types of tail behaviour. There is a type I process where variables are in the maximum domain of attraction of a Gumbell distribution. Here the tail declines exponentially and all the moments of the distribution are bounded. Examples of this process include the commonly used normal and log normal distributions where the fast tail decline results in thin tailed distributions. Furthermore, there is a type III process where variables are in the maximum domain of attraction of a Weibull distribution. Here there is no tail defined in the sense that there is no observed values beyond a certain threshold at the end of a distribution, of which the uniform and beta processes are usually cited examples. 
In contrast, the fat tailed Fréchet distribution follows a type II process where the tail has a power decline resulting in all moments not necessarily being defined:

Type II (Fréchet): $\quad \mathrm{F}(-\mathrm{r})=\quad=\exp (\mathrm{r})^{-\alpha}$

where $1 / \alpha$ is the value of the tail index and is assumed positive.

The power decline of a type II process induces a relatively slow decay for convergence at the limit vis-à-vis the relatively faster decline of the type I process with the stable paretian and student-t processes representing specific cases. The tail index, $1 / \alpha$, can take on a number of interpretations if an underlying distribution is assumed for the random variable (returns) being analysed. For instance, if the data belongs to the family of stable paretian distributions, $1 / \alpha$ is a measure of the characteristic exponent of the stable distribution, whereas, for the student-t distribution, $1 / \alpha$ is a measure of the number of degrees of freedom. Equation (5) indicates that for fat-tailed distributions, an estimate of the tail index, $1 / \alpha$, is used to develop risk measures such as VaR computations and their inferences.

All fat tailed distributions have a common characteristic by having a regular variation at infinity property:

Type II (Fréchet): $\quad \lim \quad \underline{\mathrm{F}(-\mathrm{tr})}=\mathrm{r}^{-\alpha}$

for $\alpha>0$.

$\mathrm{t} \rightarrow \infty \quad \mathrm{F}(-\mathrm{t})$

This is the sufficient and necessary condition for fat-tailed distributions to hold (Feller, 1971). Assuming that (5) holds, and the random variable under consideration is fat tailed, then a first order approximation of its tail distribution is given as:

$\mathrm{P}\{\mathrm{R}>\mathrm{r}\} \cong \operatorname{ar}^{(-\alpha)}, \quad \mathrm{a}>0, \alpha>0$

Where $1 / \alpha$ equals the number of unbounded moments, and a is a scaling constant. 
A more detailed parametric form of the tail for all fat tailed type distributions can be obtained by taking a second order expansion:

$\mathrm{F}(-\mathrm{r})=1-\operatorname{ar}^{(-\alpha)}\left[1+\mathrm{br}^{-\beta}+0\left(\mathrm{r}^{-\beta}\right)\right], \quad \beta>0$, as $\mathrm{r} \rightarrow \infty$

$\beta$ and $b$ are second order equivalents to $\alpha$ and a.

From (8), a related tail index estimator can be developed based on order statistics so that the tail values are compared to some threshold value, $\mathrm{m}$.

Kearns and Pagan (1997) find that the moments based Hill (1975) estimator dominates other approaches, and in particular, the Hill index does not suffer from small sample bias and is relatively more efficient. The Hill estimator uses order statistics as opposed to averages:

$\hat{\gamma}=1 / \alpha=(1 / m) \sum\left[\log R_{(n+1-i)}-\log R_{(n-m)}\right] \quad$ for $\mathrm{i}=1 \ldots . m$

where $R_{i}$ are the lowest (negative) order statistics. The tail estimator is asymptotically normal, $(\gamma-\hat{\gamma}) /(\mathrm{m})^{1 / 2} \approx\left(0, \gamma^{2}\right)$. The Hill estimator represents a semi-parametric measure detailing tail behaviour only rather than examining the full distribution of returns using the inferior fully parametric approaches. For the VaR analysis, it is necessary to understand the maximum loss for a specified period, but also, it is advantageous to determine if there is stability in this measure. This would allow us to extend the analysis from downside risk measures to upside risk measures (dealing with short positions). Tail stability testing is based on a Loretan and Phillips (1994) test statistic:

$\mathrm{V}\left(\gamma^{+}-\gamma^{-}\right)=\left[\gamma^{+}-\gamma^{-}\right]^{2} /\left[\gamma^{+2} / \mathrm{m}^{+}+\gamma^{-2} / \mathrm{m}^{-}\right]^{1 / 2}$

for $\gamma^{+}\left(\gamma^{-}\right)$is the estimate of the right (left) tail, and the statistic asymptotically follows a $\chi^{2}$ distribution. 
Using (9) to determine the tail index, various VaR quantile estimates can be generated using the following:

$\mathrm{q}_{\mathrm{t}}=\mathrm{R}_{(\mathrm{m})}(\mathrm{m} / \mathrm{nt})^{\gamma}$

This allows the development of $\mathrm{VaR}$ type statements. The quantiles measured by (11) shows the maximum loss for different confidence levels. It can be used to determine the extent to which a negative return exceeds the VaR threshold point. For example, a resulting statement might be as follows: the Value at Risk threshold point is exceeded every $\mathrm{x}$ days. A related measure requiring the calculation of the tail index gives different probabilities of having negative returns in excess of the threshold, the excess loss probability estimator. The downside risk statement in this case might read as follows: there is an $\mathrm{x} \%$ probability of having negative returns in excess of some loss level y\%. The excess loss probability calculation is based on the following:

$\mathrm{p}=\left(\mathrm{R}_{(\mathrm{m})} / \mathrm{R}_{\mathrm{p}}\right)^{1 / \gamma} \mathrm{m} / \mathrm{n}$

\section{DATA CONSIDERATIONS}

Characteristics for the full distribution of European equity index returns are presented. The data is obtained from Datastream for the period between the beginning of 1998 to the end of April 1999, representing 2934 returns. These returns are calculated by the first difference of the natural logarithms of equity prices. Five equity indexes from separate European markets are included for analysis. These indexes are as follows: ISEQ (Ireland), FTSE100(UK), CAC40 (France), DAX100 (Germany), and IBEX35 (Spain). The criteria used to choose 
the indexes are to examine a representative sample of European exchange traded equities based on different size classifications, and the provision of available and sufficient data for each index. For example, The ISEQ and IBEX35 indexes come from smaller exchanges (Dublin and Madrid) with an associated dearth in trading activity. In contrast, the DAX100 and FTSE100 indexes are based on the trading activity of much larger exchanges (Frankfurt and London) and would involve relatively active trading volumes. Lodged between these is the CAC40 index, which comes from a medium sized exchange (Paris) in the context of the European equity markets. In terms of the number of returns examined the aim is to examine the common longest time period possible for which data is available for each index. The number of returns used for each index also represents a sufficient number of observations to complete the tail analysis. Furthermore, the choice of European indexes ensures inclusion of a representative sample of portfolio proxies for these exchanges over the last decade. ${ }^{5}$

Summary Statistics are shown in table 1, where details of the first four moments, the mean, standard deviation, skewness and centred kurtosis are presented. Generally, these statistics show similar characteristics for the equity indexes, namely, that daily returns on average are positive, risk levels are around one percent per day, there is excess negative skewness (FTSE100 excepted), and the returns are leptokurtotic. This latter finding is the driving force behind the application of extreme value theory and the assumption that the equity index returns are in the maximum domain of attraction of the fat tailed, Fréchet distribution. Also, both third and fourth moment values are evidence against the normal distribution at conventional probability levels. This issue is formally examined using the 
Kolmogorov-Smirnov test Normality is formally examined with the KolmogorovSmirnov test indicating that none of the indexes support the hypothesis of belonging to a gaussian distribution. These findings are similar to previous studies on equity markets. This non-normality suggests the ruling out of the mean-variance framework applied in portfolio theory as a method of measuring the downside risk estimates. In fact assuming normality results in an underestimation of tail behaviour and by extension the associated downside risk measures. As this study concentrates on extreme values for the downside risk measures statistics are also given for maximum and minimum values, as well as the first and third quantiles. Again the indexes show similar values for the period analysed, of which the DAX100 outliers shows the greatest range, and CAC40 has the largest interquartile range. Notwithstanding the findings in table 1 however, these summary statistics indicate that further analysis is required to get realistic outcomes on the downside risk exposures facing investors on these exchanges.

\section{INSERT TABLE 1 HERE}

It is interesting to investigate further the more extreme downside return values given the findings in table 1. Taking the DAX100 index as a case study (as this portfolio experiences the greatest magnitude of losses), the lowest one percent of equity returns and their time of occurrence are presented in table 2 . The beginning and end of the sample period represent the highest negative extreme returns with very few observations during the middle of the period of analysis. The extent of the magnitude of the losses suffered in Frankfurt is evident by noting that the worst sixteen returns in table 2 dominate the worst recorded return on the London exchange as shown in table 1. There is no evidence of clustering of extreme returns 
unlike the general findings from the analysis of the full distribution of financial return datasets.

\section{EMPIRICAL FINDINGS}

Empirical findings of the Hill tail index for each equity index are now discussed. The lower tail estimates are given in table 3 for two threshold values of one and five percent, both in the spirit of the DuMouchel (1983) technique, but also corresponding to the most commonly cited confidence levels. There are a number of interesting findings. To begin, the tail index values range from 2.44 (DAX100) to 5.57 (FTSE100) for the analysis of all tail estimates. Concentrating specifically on the downside tail estimates we see that the upper tail estimate is 4.37 (FTSE100). These values correspond to the general conclusions made for time series of financial returns, namely that they have fat-tail characteristics. The goodness of fit of each equity index being associated with the Fréchet distribution is confirmed in table 2 using a difference in means statistic given in Koedijk and Kool (1992). Specifically all tail values are significantly positive, corresponding to the requirement that $\gamma=\alpha^{-1}>0$.

Previous studies on financial returns have classified the data according to different fat-tailed distributions on the basis of the tail estimates obtained. We can characterise Hill estimates with a value less than or equal to two as belonging to a stable paretian family of distributions, whereas, for values greater than two, they belong to GARCH related specifications (Ghose and Kroner, 1995). Formally, this is tested using the difference in means statistic for a number of hypotheses 
including belonging to the stable paretian family of distributions $\left(\mathrm{H}_{0}: \gamma<2\right)$, and having GARCH characteristics $\left(\mathrm{H}_{0}: \gamma>2\right)$. Conclusions from the downside tail estimates indicate that there is support for the stable paretian hypothesis being rejected for all of the equities with the exception of the ISEQ and IBEX35 indexes as the null $\left(\mathrm{H}_{\mathrm{o}}: \gamma^{-}<2\right)$ is statistically rejected. In contrast, the indication that the tail returns have GARCH features is never rejected.

\section{INSERT TABLE 3 HERE}

The tail estimates generally decrease as you move away from the one percent threshold with the exception of the DAX100 index. Decreases in the estimates as you move towards the centre of the distribution reaffirms the idea that the most extreme returns have different distributional characteristics than the full sequence of returns as suggested in the earlier data description. Although the main discussion of this paper is on downside risk encompassing a long position, it is easy to extend the analysis to determine upside risk focusing on a short position, and a common position regardless of the source of risk. In table 3 , upside, $\gamma^{+}$, and common, $\gamma^{*}$, tail estimates are presented. Using Loretan and Phillips (1994) stability test we can conclude that our inferences on the $\mathrm{VAR}_{\mathrm{q}}$ and $\mathrm{EL}_{\mathrm{p}}$ estimates are equally appropriate for a short position with all lower tail estimates insignificantly different from upper tail measures.

Turning to the VaR quantiles in table 4 both in-sample and out-of-sample estimates are examined. For the two thresholds, m, we examine six probability levels, either 30 in $2934\left(\mathrm{q}_{(0.01)}\right)$, or 15 in $2934\left(\mathrm{q}_{(0.005)}\right)$, or 3 in $2934\left(\mathrm{q}_{(0.001)}\right)$, or 1 in $2934\left(\mathrm{q}_{(1 / \mathrm{n})}\right)$, 
or 1 in $5868\left(\mathrm{q}_{(1 / 2 \mathrm{n})}\right)$, or 1 in $8802\left(\mathrm{q}_{(1 / 3 \mathrm{n})}\right)$, representing the expected number of occurrences. The latter two probability levels represent out-of-sample estimates detailing over 22 years and 33 years of return observations. These estimates could not be obtained using the empirical distribution as we could only deal with probabilities of $1 / \mathrm{n}$ or larger. However, extreme value theory allows for semiparametric estimation so very rare events outside of the sample size under analysis can be examined. Hence, these VaR quantile estimates could not be obtained using methods such as historical simulation due to a lack of data. ${ }^{6}$ The VaR quantiles are easy to interpret, for instance there is a 99 percent confidence that the downside risk for the ISEQ index is less than or equal to $2.43 \%$ with a tail threshold, m, of $1 \%$. Losses in excess of this value would occur with a frequency of 1 in 100 days $(n=$ $1 / \mathrm{p}$ ) on average. The robustness of the $\mathrm{VaR}$ values is verified using the technique outlined in Danielsson and de Vries (2000) comparing estimated to expected occurrences.

\section{INSERT TABLE 4 HERE}

To begin, we see that the indexes exhibit reasonably similar tail volatility for high probability levels with DAX100 exhibiting the highest (3.37\% and 3.27\%) VaR quantiles for both thresholds in comparison to the FTSE100 exhibiting the lowest (2.27\% and $2.20 \%) \mathrm{VaR}$ quantiles. ${ }^{7}$ However, by moving out the tail to more extreme quantiles we find two notable differences, namely, that the levels of volatility increase substantially and that these increases in tail volatility become dissimilar across stock indexes. On the former issue for instance, we see that the VaR quantile increases for the FTSE100 index from $2.27 \%$ to $6.33 \%$ going from in- 
sample to out-of-sample estimates. This is to be expected given the movement further out the tail to more volatile returns. Whereas on the latter issue, the out-ofsample estimates vary substantially at the tail threshold, $\mathrm{m}$, of 5\% (the variation becomes even more pronounced for the $1 \%$ tail threshold, m) with the DAX100 now exhibiting downside risk of less than or equal to $15.85 \%$ in comparison to $6.33 \%$ for the FTSE100. This provides an insight into the respective volatility of each individual index that would not normally be presented with more commonly used statistical measures.

The excess loss probability estimators are presented in table 5 where both in-sample and out-of-sample estimates are examined. Again we examine two thresholds, and provide a large number of extreme value estimates for the downside risk of the European indexes. Five percentage quantiles are presented and we determine the probability of each index exhibiting the sufficient volatility to be in excess of these quantiles. These quantiles are (negative) $10 \%\left(\mathrm{P}_{(0.1)}\right), 5 \%\left(\mathrm{P}_{(0.05)}\right), 1 \%\left(\mathrm{P}_{(0.01)}\right), 0.5 \%$ $\left(\mathrm{P}_{(0.005)}\right)$, and $0.1 \%\left(\mathrm{P}_{(0.001)}\right)$. The excess loss probability estimators imply that the probability on any given day of having a negative return exceeding $10 \%$ for the ISEQ index is $0.02 \%$ with a tail threshold, $\mathrm{m}$, of $1 \%$. Any value in excess of 100 relates to the out-of-sample occurrence of an event as we are certain that that percentage quantile will be exceeded in the time frame analysed and would have a likelihood of occurrence in an extended time period.

INSERT TABLE 5 HERE 
As we can see in table 5, there is a very low probability of the respective indexes exceeding large losses such as $10 \%$. For instance, the most risky asset at this quantile of the tail is the DAX100 index at the $1 \%$ threshold, m, with an associated probability of $0.07 \%$. However, as you move to smaller losses, the associated probability levels increase as expected a priori that this negative relationship occurs. To illustrate, we see that there is a $100 \%$ likelihood that each index will incur losses in excess of $0.1 \%$. Obviously such price movements are minimal in the context of observed daily volatility for risky assets such as equities and these are expected to definitely occur. Returning to larger losses such as $1 \%$, we see for example that there is a $36.19 \%$ probability of downside returns being in excess of this loss level for the FTSE100 index. This can be measured in terms of the actual numbers of days (given the sample size) that the London traded index exceeds these loss levels with occurrences of 1062 days as given by the calculation $n^{*} \mathrm{p}$.

As stated the DAX100 index exhibits the most volatility around the very large loss levels such as $10 \%$ or even $5 \%$. However as you move to more common (or smaller) loss levels we see that the other indexes indicate more volatility, with for example both the FTSE100 and CAC40 indexes having a greater probability of exceeding losses of $0.1 \%$ at the $1 \%$ threshold, $\mathrm{m}$. The rational for this divergence at different loss levels can be explained in terms of relative fatness of the tail and the relative scale of the tail values. Generally, we can conclude that the FTSE100 and CAC40 index have fatter tails than the DAX100 index with their higher propensity to exceed the lower loss levels. In comparison the relatively thin tailed DAX100 contract at these lower loss levels has extreme values of greater magnitude. These relatively large extreme values impact the probability estimates 
in the second column of table 5. Further evidence supporting this rational can be found in terms of the magnitude of the extreme values for this index presented in table 4 for all VaR quantiles and in table 2 with the largest minimum value.

\section{CONCLUSIONS}

This paper applies extreme value theory to measure downside risk for European equity markets. Two related risk measures are presented, the popular Value at Risk estimator, and the Excess Loss probability estimator. Overall, these measures allow for a coherent risk management approach to optimally protect investor wealth opportunities. Given the large fluctuations inherent in equity markets, the empirical application examines combinations of low quantile and probability estimates. Whilst these downside events may not occur with everyday regularity, their consequences demand a thorough analysis. Fortunately, the extreme value approach focuses on tail price fluctuations at the expense of the whole distribution, as it is these outcomes that affect the relative ability for an investor's portfolio to survive in the face of large price movements.

Extreme value theory dominates alternative approaches in tail estimation as it avoids model risk. Alternative approaches such as the variance covariance and Monte Carlo methods model the full distribution of returns, whereas this study's focus of attention, the tail values are explicitly modelled by extreme value theory. Also, extreme value theory operates on the basis that the regular variation at infinity property is the necessary and sufficient condition for fat-tailed distributions to hold thereby inducing an identical limiting feature. This property removes the need to 
exactly match any set of returns exhibiting a fat-tailed characteristic with a particular distributional form. Moreover, the theoretical approach adopted here advantageously can be extended for different frequencies and for out-of-sample estimates using parsimonious techniques. The former relies on a simple scaling law whereas the latter exploits the semi-parametric estimation technique to extend beyond a given sample size. On the issue of semi-parametric estimation, the paper utilises the moments based Hill estimator, which dominates other tail estimators on bias and efficiency grounds.

Notwithstanding the agreement with general findings of excess skewness, leptokurtosis and a lack of normality cited for equity markets, this paper makes a number of interesting findings. First, all equity series' statistically belong to the Fréchet distribution, and more specifically exhibit GARCH characteristics at the expense of the stable paretian model. Second, whereas the VaR quantiles increase from in-sample to out-of-sample estimates, the degree of these increases vary according to each index. In particular, the DAX100 index exhibits comparably higher downside risk levels demonstrating this portfolio's unique volatility pattern identified by extreme value analysis. Third, the excess probability estimates provide further evidence of the implications of the respective fat-tailed characteristics of each index. Here, we find that the FTSE100 and CAC40 indexes differ from the DAX100 series in that they have a higher propensity for exceeding relatively small losses but a lower propensity for exceeding relatively large losses. Finally, we conclude that all our inferences on the downside VaR quantile and excess probability estimates hold for the upside due to the stability of returns located at the upper tail of the indexes' distribution. 


\section{ACKNOWLEDGEMENTS}

The author would like to thank participants at the Economics Department seminar, University College Cork for their helpful comments on this paper. Financial funding was given by the President's research award, University College Dublin. 


\section{REFERENCES}

Artzner, P., DelBaen, F., Eber, J. and Heath, D. (1999) Coherent measures of risk, Mathematical Finance, 8, 203-228.

Arzac, E. R., and Bawa, V. S. (1977) Portfolio choice and equilibrium in capital markets with safety-first investors, Journal of Financial Economics, 4, 277-288.

Cotter, J. (2001) Margin exceedences for european stock index futures using extreme value theory, Journal of Banking and Finance, 25, 1475-1502.

Danielsson, J and DeVries, C. G. (1997a) Tail index and quantile estimation with very high frequency data, Journal of Empirical Finance, 4, 241-257.

Danielsson, J and DeVries, C. G. (1997b) Beyond the sample: extreme quantile and probability estimation, Mimeo, Timbergen Institute Rotterdam, December.

Danielsson, J and DeVries, C. G. (2000) Value at Risk and extreme returns, Annales D'Economie et de Statistique, 60, 239-270.

Diebold, F. X., Schuermann, T. and Stroughair, J. D. (1998) Pitfalls and opportunities in the use of extreme value theory in risk management, in Advances in Computational Finance, (Editors) J. D. Moody and A. N. Burgess, Kluwer, Amsterdam.

Duffie, D., and Pan, J. (1997) An overview of value at risk, Journal of Derivatives, 4, 7-49.

Feller, W. (1971) An introduction to probability theory and its applications, John Wiley, New York.

Gallagher, L. A. (1999) A multi-country analysis of the temporary and permanent components of stock prices, Applied Financial Economics, 9, 129-142. 
Ghose, D., and Kroner, K. F. (1995), The relationship between garch and symmetric stable processes: finding the source of fat tails in financial data, Journal of Empirical Finance, 2. 225-251.

Hill, B. M. (1975) A simple general approach to inference about the tail of a distribution, Annals of Statistics, 3, 1163-1174.

Jansen, D. W., Koedijk, K. G. and DeVries, C. G. (2000) Portfolio selection with limited downside risk, Journal of Empirical Finance, 7, 247-269.

Kearns, P. and Pagan, A. (1997) Estimating the density tail index for financial time series, The Review of Economics and Statistics, 79, 171-175.

Koedijk, K. G. and Kool, C. J. M. (1992) Tail estimates of east european exchange rates, Journal of Business and Economic Statistics, 10, 83-96.

Leadbetter, M. R., Lindgren, G. and Rootzen, H. (1983) Extremes and related properties of random sequences and processes, Springer, New York.

Loretan, M. and Phillips, P. C. B. (1994) Testing the covariance stationarity of heavy-tailed time series, Journal of Empirical Finance, 1, 211-248.

Poon, S. H., and Taylor, S. (1992) Stock returns and volatility: an empirical study of the UK stock market, Journal of Banking and Finance, 45, 37-59.

Venkataraman, S. (1997) Value at Risk for a mixture of normal distributions: the use of quasi-bayesian estimation techniques, Federal Reserve Bank of Chicago's Economic Perspectives, March/April, 2-13. 
Figure 1: Value at Risk for a downside quantile of the distribution of returns

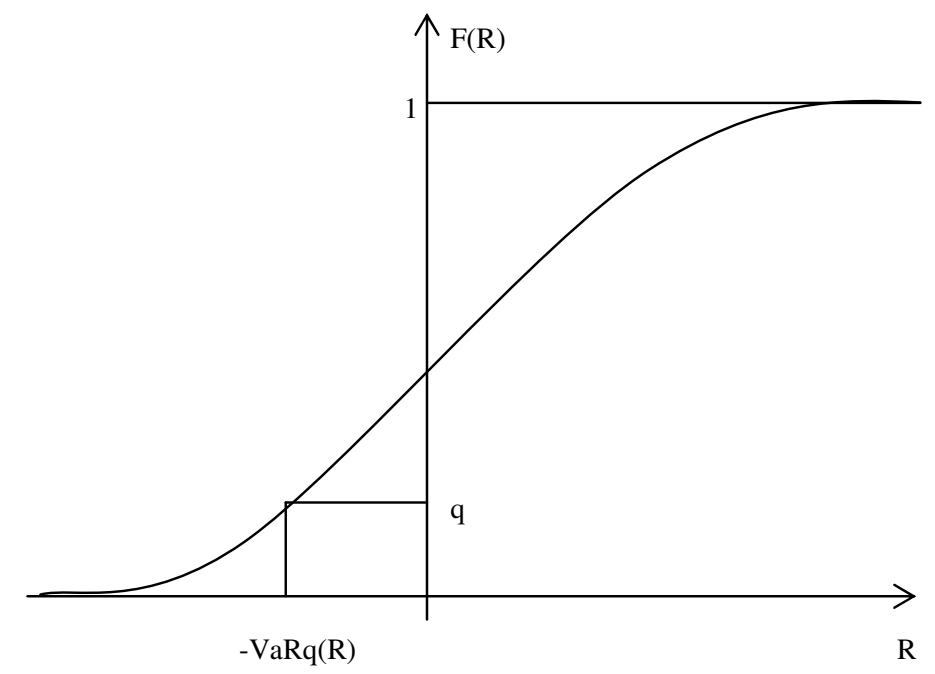


Figure 2: Left tail of fat-tailed and normal distribution

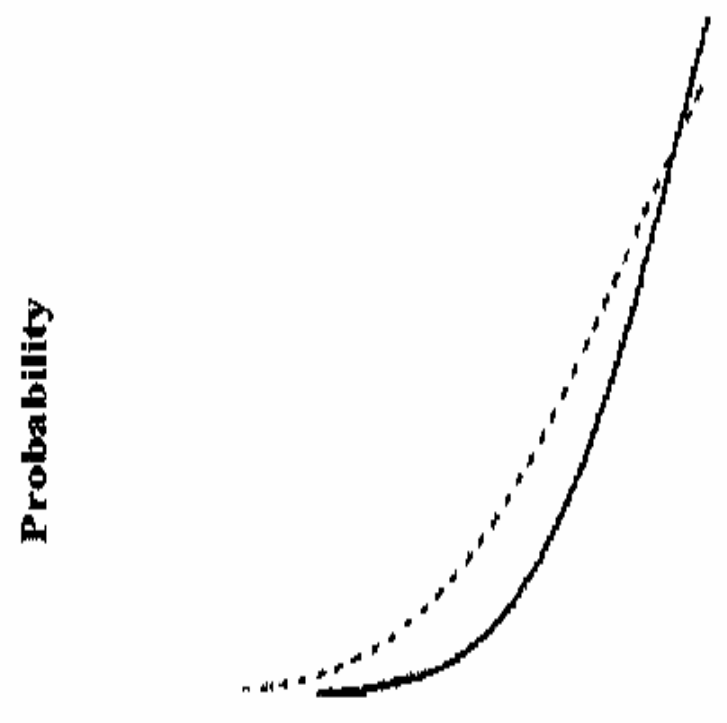

The fat-tailed distribution (shaded curve) is plotted against the normal distribution (solid curve). 
Table 1: Summary Statistics of Equity Index Returns

\begin{tabular}{llllllllll}
\hline Series & Mean & Max & Min & $\begin{array}{c}\text { Standard } \\
\text { Deviation }\end{array}$ & Q1 & Q3 & Skew & Kurt $^{\text {a }}$ & Normality \\
\hline ISEQ & 0.057 & 6.041 & -7.569 & 0.898 & -0.366 & 0.476 & $-0.274^{*}$ & $8.227^{*}$ & $0.076^{*}$ \\
FTSE100 & 0.045 & 5.440 & -4.140 & 0.869 & -0.473 & 0.564 & 0.500 & $2.357^{*}$ & $0.031^{*}$ \\
CAC40 & 0.049 & 5.808 & -7.573 & 1.178 & -0.596 & 0.723 & $-0.172^{*}$ & $2.983^{*}$ & $0.050^{*}$ \\
DAX100 & 0.045 & 8.659 & -14.055 & 1.178 & -0.501 & 0.660 & $-0.872 *$ & $14.306^{*}$ & $0.074^{*}$ \\
IBEX35 & 0.048 & 6.837 & -8.876 & 1.169 & -0.501 & 0.640 & $-0.402 *$ & $6.358^{*}$ & $0.074 *$
\end{tabular}

The summary statistics are presented for each stock index returns. The mean, minimum and maximum values represent the average, lowest and highest returns respectively. The first, Q1, and third quartile, Q3, represent the $25^{\text {th }}$ and $75^{\text {th }}$ percentiles. These five measures are presented in percentage form. The skewness (Skew) statistic is a measure of distribution asymmetry with symmetric returns having a value of zero. The centred kurtosis (Kurt) statistic measures the shape of a distribution vis-à-vis a normal distribution with a gaussian density function having a value of zero. Normality is formally examined with the Kolmogorov-Smirnov test which indicates a gaussian distribution with a value of zero. The symbol * represents significant at the $5 \%$ level. 
Table 2: Dates and Lowest One Percent Extreme Values for DAX100 index Returns

\begin{tabular}{llll}
\hline Date & \multicolumn{1}{l}{$(\%)$ Return Date } & $(\%)$ Return \\
\hline $13 / 10 / 89$ & -14.05 & $24 / 09 / 90$ & -4.32 \\
$16 / 08 / 91$ & -10.06 & $24 / 10 / 97$ & -4.04 \\
$27 / 10 / 97$ & -8.11 & $21 / 08 / 97$ & -3.93 \\
$19 / 08 / 96$ & -7.24 & $05 / 12 / 96$ & -3.84 \\
$01 / 10 / 98$ & -6.14 & $10 / 08 / 98$ & -3.81 \\
$01 / 01 / 88$ & -5.67 & $16 / 09 / 98$ & -3.81 \\
$20 / 08 / 98$ & -5.59 & $11 / 01 / 91$ & -3.67 \\
$03 / 08 / 90$ & -5.59 & $31 / 03 / 97$ & -3.56 \\
$09 / 09 / 98$ & -5.48 & $22 / 08 / 90$ & -3.53 \\
$30 / 09 / 98$ & -5.41 & $17 / 06 / 94$ & -3.43 \\
$20 / 08 / 90$ & -5.16 & $08 / 01 / 88$ & -3.42 \\
$12 / 01 / 99$ & -4.92 & $16 / 08 / 90$ & -3.42 \\
$07 / 10 / 98$ & -4.62 & $18 / 09 / 98$ & -3.40 \\
$30 / 11 / 98$ & -4.44 & $09 / 01 / 98$ & -3.39 \\
$22 / 10 / 97$ & -4.36 & $02 / 10 / 92$ & -3.36 \\
\hline
\end{tabular}


Table 3: Tail Index Estimates for European Stock Indexes

\begin{tabular}{|c|c|c|c|c|c|}
\hline Series & $\gamma^{+}$ & $\gamma$ & $\gamma^{*}$ & $\gamma^{+}=\gamma^{-}$ & $\gamma=2$ \\
\hline \multicolumn{6}{|l|}{$(\mathrm{m}=1 \%)$} \\
\hline & 3.26 & 2.77 & 3.67 & 0.63 & 1.51 \\
\hline ISEQ & $(0.60)$ & $(0.51)$ & $(0.67)$ & & \\
\hline \multirow[t]{2}{*}{ FTSE100 } & 4.10 & 4.37 & 5.57 & -0.25 & $2.96^{\circ}$ \\
\hline & $(0.75)$ & $(0.80)$ & $(1.02)$ & & \\
\hline \multirow[t]{2}{*}{ CAC40 } & 3.64 & 4.02 & 4.32 & -0.38 & $2.85^{\circ}$ \\
\hline & $(0.66)$ & $(0.73)$ & $(0.79)$ & & \\
\hline \multirow[t]{2}{*}{ DAX100 } & 2.55 & 2.89 & 2.60 & -0.48 & $1.68^{\circ}$ \\
\hline & $(0.47)$ & $(0.53)$ & $(0.47)$ & & \\
\hline \multirow[t]{2}{*}{ IBEX35 } & 3.28 & 2.66 & 3.61 & 0.81 & 1.35 \\
\hline & $(0.60)$ & $(0.49)$ & $(0.66)$ & & \\
\hline \multicolumn{6}{|l|}{$(\mathrm{m}=5 \%)$} \\
\hline \multirow[t]{2}{*}{ ISEQ } & 3.00 & 2.60 & 2.85 & 1.21 & $2.86^{\circ}$ \\
\hline & $(0.25)$ & $(0.21)$ & $(0.24)$ & & \\
\hline \multirow[t]{2}{*}{ FTSE100 } & 3.18 & 3.07 & 3.13 & 0.31 & $4.28^{\circ}$ \\
\hline & $(0.26)$ & $(0.25)$ & $(0.26)$ & & \\
\hline \multirow[t]{2}{*}{ CAC40 } & 3.41 & 2.92 & 3.70 & 1.30 & $3.83^{\circ}$ \\
\hline & $(0.28)$ & $(0.24)$ & $(0.31)$ & & \\
\hline \multirow[t]{2}{*}{ DAX100 } & 2.85 & 2.44 & 2.70 & 1.32 & $2.20^{\circ}$ \\
\hline & $(0.24)$ & $(0.20)$ & $(0.22)$ & & \\
\hline \multirow[t]{2}{*}{ IBEX35 } & 3.04 & 2.58 & 3.04 & 1.39 & $2.76^{\circ}$ \\
\hline & $(0.25)$ & $(0.21)$ & $(0.25)$ & & \\
\hline
\end{tabular}

Tail estimates, $\gamma=1 / \alpha$, are calculated for lower, upper and both tails for each stock index. The symbols,,$-+ *$ represent the lower, upper and both tails respectively. The threshold values, $\mathrm{m}$, relate to the one $(\mathrm{m}=30)$ and five $(\mathrm{m}=147)$ percentiles. Standard errors are presented in parenthesis for each tail value. Tail stability is calculated in the second last column. The lower tail values are compared to 2 in the last column with the symbol $\bullet$ representing significance at a $5 \%$ level. 
Table 4: Quantile Estimates for European Stock Indexes

\begin{tabular}{|c|c|c|c|c|c|c|}
\hline \multirow[t]{2}{*}{ Series } & $\mathrm{q}_{(0.01)}$ & $\mathrm{q}_{(0.005)}$ & $q_{(0.001)}$ & $\mathrm{q}_{(1 / \mathrm{n})}$ & $\mathrm{q}_{(1 / 2 \mathrm{n})}$ & $q_{(1 / 3 n)}$ \\
\hline & $30 / 2934$ & $15 / 2934$ & 3/2934 & $1 / 2934$ & $1 / 5868$ & $1 / 8802$ \\
\hline \multicolumn{7}{|l|}{$\mathrm{m}=1 \%$} \\
\hline \multirow[t]{2}{*}{ ISEQ } & 2.43 & 3.12 & 5.57 & 8.22 & 10.56 & 12.22 \\
\hline & (29) & (15) & (3) & (0) & & \\
\hline \multirow[t]{2}{*}{ FTSE100 } & 2.27 & 2.66 & 3.85 & 4.93 & 5.77 & 6.33 \\
\hline & (29) & (16) & (2) & (0) & & \\
\hline \multirow[t]{2}{*}{ CAC40 } & 3.19 & 3.79 & 5.66 & 7.39 & 8.79 & 9.72 \\
\hline & (30) & (15) & (3) & (1) & & \\
\hline \multirow[t]{2}{*}{ DAX100 } & 3.37 & 4.28 & 7.47 & 10.84 & 13.78 & 15.85 \\
\hline & (29) & (16) & (3) & (1) & & \\
\hline \multirow[t]{2}{*}{ IBEX35 } & 3.19 & 4.14 & 7.58 & 11.36 & 14.74 & 17.17 \\
\hline & $(30)$ & (17) & (3) & $(0)$ & & \\
\hline \multicolumn{7}{|l|}{$\mathrm{m}=5 \%$} \\
\hline \multirow[t]{2}{*}{ ISEQ } & 2.35 & 3.07 & 5.71 & 8.64 & 11.27 & 13.18 \\
\hline & (32) & (17) & (3) & $(0)$ & & \\
\hline \multirow[t]{2}{*}{ FTSE100 } & 2.20 & 2.76 & 4.66 & 6.62 & 8.30 & 9.47 \\
\hline & (31) & (16) & (0) & $(0)$ & & \\
\hline \multirow[t]{2}{*}{ CAC40 } & 3.17 & 4.02 & 6.97 & 10.08 & 12.78 & 14.68 \\
\hline & $(30)$ & (14) & (2) & $(0)$ & & \\
\hline \multirow[t]{2}{*}{ DAX100 } & 3.27 & 4.34 & 8.40 & 13.05 & 17.34 & 20.47 \\
\hline & (31) & (15) & (2) & (1) & & \\
\hline \multirow[t]{2}{*}{ IBEX35 } & 3.23 & 4.22 & 7.88 & 11.96 & 15.65 & 18.31 \\
\hline & (29) & (16) & (2) & (0) & & \\
\hline
\end{tabular}

The values in this table represent the $\mathrm{VaR}$ quantiles for different confidence intervals, for example $\mathrm{q}_{(0.01)}$ is the $1 \%$ level. The estimates are based on different threshold values, $\mathrm{m}$, which are used to calculate the associated lower tail estimates, $\bar{\gamma}$, for each stock index. Values are expressed in percentages. The expected number of occurrences is based on the associated probabilities, for example $30 / 2934$ is the $1 \%$ level. The estimated number of occurrences for each in-sample 
confidence level is presented in parentheses. Data unavailability precludes comparisons of lower quantile estimates. 
Table 5: Probability Estimates for European Stock Indexes

\begin{tabular}{rrrrrr}
\hline Series & $\mathrm{P}_{(0.1)}$ & $\mathrm{P}_{(0.05)}$ & $\mathrm{P}_{(0.01)}$ & $\mathrm{P}_{(0.005)}$ & \multicolumn{1}{c}{$\mathrm{P}_{(0.001)}$} \\
\hline $\mathrm{m}=1 \%$ & & & & & \\
ISEQ & 0.02 & 0.14 & 11.66 & 79.55 & 6867.52 \\
FTSE100 & 0.00 & 0.03 & 36.19 & 748.24 & 848285.58 \\
CAC40 & 0.01 & 0.16 & 106.04 & 1720.29 & 1110352.27 \\
DAX100 & 0.04 & 0.32 & 33.41 & 247.62 & 25930.71 \\
IBEX35 & 0.05 & 0.30 & 21.86 & 138.15 & 9990.71 \\
& & & & & \\
m =5\% & & & & & \\
ISEQ & 0.02 & 0.14 & 9.27 & 56.18 & 3688.91 \\
FTSE100 & 0.01 & 0.08 & 11.29 & 94.84 & 13269.02 \\
CAC40 & 0.03 & 0.26 & 29.00 & 219.49 & 24121.11 \\
DAX100 & 0.07 & 0.35 & 17.98 & 97.55 & 4951.18 \\
IBEX35 & 0.05 & 0.32 & 20.57 & 123.00 & 7820.58
\end{tabular}

The values in this table represent the probability of returns exceeding a certain threshold, for example, $\mathrm{P}_{(0.1)}$ is 10 percent on any single day. The estimates are based on different threshold values, $\mathrm{m}$, which are used to calculate the associated lower tail estimates, $\gamma$, for each stock index. Values are expressed in percentages. Any value in excess of 100 implies that the Extreme Value estimates predict with certainty the existence of such an event during an equivalent sample period. The values are included to indicate the divergence in the stock indexes tail behaviour. 


\section{FOOTNOTES}

1 This paper exclusively analyses downside risk, the outcomes of interest for a long position located at the left tail of the distribution of stock index returns. The theory utilised is equally applicable for a short position dealing with the right tail of a distribution.

$1 \mathrm{VaR}$ was originally developed as part of the Riskmetrics system produced by JP Morgan to meet the requirement of its CEO Dennis Weatherstone to have a single measure of risk exposure presented to him on a daily basis. The measure has quickly spread to become a mainstream and commonly used risk management analytical tool. However, downside quantile estimation has been applied in the past prior to the J.P.Morgan development (see Arzac and Bawa, 1977; for one such illustration of portfolio selection with an inbuilt safety criteria).

3 There may also be a regulatory need to have VaR measures over longer periods, for example, the Basle capital requirements for a ten day holding period.

4 The maximum domain of attraction does not operate in reverse, so the assumption that the mixtures of normals converges to a Fréchet distribution does not imply that the Fréchet reaslisations follow a mixtures of normals process.

5 Whilst it is recognised that an investor's diversified portfolio may not actually match any of the indexes analysed, they are representative of diversified groups of individual equities traded on the respective exchanges.

6 Using historical simulation would accentuate the problem even further if the returns series was smaller (common in many academic studies).

7 The effects of German unification are an obvious cause of these high volatility estimates. Further examination of the components of each of the indexes, for 
example industry sector analysis, may provide further indications of the source of their tail volatility. 